\title{
A sketch for a photolytic history of organic molecules in the Solar System.
}

\author{
F.R. ORTHOUS-DAUnAY, ${ }^{1}$ L. Piani, ${ }^{2}$ L. FLANDINET, ${ }^{1}$ R. \\ Thissen, ${ }^{3}$ C. Wolters, ${ }^{1}$ V. Vuitton, ${ }^{1}$ O. PoCh,${ }^{1}$ F. \\ MOYNIER, ${ }^{4}$ I. SUGAWARA, ${ }^{5}$ H. NARAOKA ${ }^{6}$ AND S.
} TACHIBANA $^{5,7}$

${ }^{1}$ IPAG, Univ. Grenoble Alpes, Grenoble, France, frod@univ-grenoble-alpes.fr ${ }^{2} \mathrm{CRPG}$, Vandoeuvre-lès-Nancy, France ${ }^{3}$ ICP, Université Paris-Saclay, Orsay, France ${ }^{4}$ IPGP, Univ Paris Diderot, Paris, France ${ }^{5}$ DNHS, Hokkaido University, Sapporo, Japan ${ }^{6}$ DEPS, Kyushu University, Fukuoka, Japan ${ }^{7}$ UTOPS, University of Tokyo, Tokyo, Japan

Introduction: The Soluble Organic Matter (SOM) consists of thousands of compounds [1], [2]. Their size is in-between the molecules detected in space (tens of atoms) and macromolecules found only in meteorites and can be related to their synthesis process [3], [4]. The carbonaceous chains size distributions is consistent with an interstellar origin. Recent studies reported efficient aliphatic chains growth in hot corinos [5]. An experimental test of this origin would be to expose SOM to photolytic condition and to monitor any chemical change with high resolution MS.

Method: Sixty-five g of Murchison were disaggregated before being macerated in methanol and toluene. Thin films of extract were deposited on sapphire glasses and exposed to UV (115 to $165 \mathrm{~nm})$ deuterium lamps under $\sim 10^{-6} \mathrm{~Pa}$ vacuum inside the PICACHU [6,7] apparatus at Hokkaido University. Samples received various fluencies of photons, reaching $10^{20}$ photons $/ \mathrm{cm}^{2}$ in 500 hours of exposure. Chemical modification was checked by high resolution MS before and after exposure with a Thermo LTQ Orbitrap XL, at Univ. Grenoble Alpes.

Results and discussion: Ions are in the 150-750 Da range without holes in their distribution. In this mass range, the Orbitrap resolution is high enough for stoichiometry computation. We interpret the periodicity in mass as a repetition of stoichiometric patterns. Molecules varying only by a given number of $\mathrm{CH}_{2}$ all exhibit a lognormal distribution. This is characteristic of chains transfer polymerization. After exposure, we observe a lognormal distribution but with significant variations of the distribution parameters.

References: [1]Schmitt-Kopplin P. et al. (2010) Proc. Natl. Acad. Sci. U. S. A., 107, 7 pp. 2763-8.[2]Yamashita Y. and Naraoka H. (2014) Geochem. J., 48 pp. 519-525.[3]Wesslau H. (1956) Makromol. Chem., 20 p. 111.[4]Somogyi Á. et al. (2016) Int. J. Mol. Sci., 17, 439.[5]Belloche A. et al. (2014) Science (80-. )., 345, 6204 p. 15841587.[6]Piani L. et al. (2017) Astrophys. J. 837, 12 pp.[7]Tachibana S. et al. (2017) Sci. Adv. 3, 6 pp. 